\title{
The Case for Pharmacy Report Cards
}

\author{
Report cards, important measures of health plan and provider \\ performance, exist for every segment of the health care team- \\ except pharmacists.
}

\section{Jerry E. Silverman and Teri R. Rosen}

\section{Hintor}

JERRY E. SILVERMAN, R.P.H., is President, and TERI R. ROSEN; M.A., is Vice President of Program Development, Bimark Healthcare Communications, Hackensack, NJ.

Copyright $(1$ 1999, Academy of Managed Care Pharmacy, Inc. All rights reserved.
$\mathrm{T}$ oday's employers hold health plans accountable for both price control and quality measures. As purchasers of health care, employers increasingly strive to acquire the best quality health care available within their budgets. Service that keeps employees healthy and productive is more cost effective over the long run, resulting in fewer days lost from work due to illness or disability.

Employer interest in service quality has increased as health care costs have risen exponentially. By 2000 , annual per capita spending for health care is expected to reach $\$ 4,700$, accounting for almost $20 \%$ of consumer spending. Over the last 40 years, health care expenditures have grown at an annual rate of almost $10 \%$. In contrast, over the same time period, annual personal and corporate income has increased by less than $5 \% .^{1}$ To justify this exorbitant escalation, health plans have begun to gather data on quality of care so that they can substantiate their worth to corporate purchasers, patients, and the public at large.

Yet the health care industry spends significantly less on information technology than do other large industries. The banking industry, for example, spends $6 \%$ of total revenue on gathering and storing information on its operations; health care plans, whose need for accurate information is no less acute, spend less than $1.5 \%$ of total revenue on the collection and study of outcomes information. Part of the explanation may lie in the technology limitations that plans currently face: Accumulating outcomes data would far exceed most health care " plans' outcomes-measurement and datamanagement capacities at this time. ${ }^{2}$

Report cards are credible, objective instruments for collecting and commu- nicating comparative information about the performance of health plans. They have evolved to meet employers' demands for quantitative and qualitative documentation of the value of health care services. The ideal health care report card covers capabilities/service requirements, patient satisfaction measures, and outcome markers such as the rate of generic substitution and compliance with selected medical therapies for costly chronic diseases (e.g., hypertension, hyperlipidemia, and depression).

The shortage of outcomes data in the late 1980s prompted a few large employers to seek comparative statistics on health maintenance organization (HMO) or managed care organization (MCO) performance. As the demand for information grew over the next few years, the National Committee for Quality Assurance began developing the standard for today's report cards - the Health Plan Employer Data and Information Set (HEDIS).

HEDIS is a continually evolving instrument that provides both consumers and health care purchasers with comprehensive and meaningful information on health plan performance. As part of this evolution, outcomes-based report cards have been developed for every segment of the health care team-hospitals, health care plans, and health care professionals-except pharmacists. Such an exception has become untenable, given the increasing utilization and cost of prescription drugs that are compelling employers to evaluate the quality of their pharmacy benefits. One important. criterion in such an evaluation is the quality of a network's pharmacy providers. Pharmacy report cards would furnish employers, MCOs, and pharmacy 
Continued from page 176

benefit managers (PBMs) with reliable information that could be used to improve the quality of their networks.

\section{PHARMACEUTICAL CARE}

Pharmacy accounts for an increasing percentage of total health care dollars. The pharmacist has become a key member of the interdisciplinary health care team because pharmacists are positioned to have the greatest impact on the appropriate use of medications.

The role of the pharmacist is to improve patient outcomes by optimizing. pharmacotherapeutic regimens. Pharmacists are trained to view people not as customers purchâsing a product but as patients in need of care. Pharmaceutical care begins with patient/pharmacist face-to-face contact and communication. One measure of effective pharmaceutical care is the extent to which patients comply with prescribed medical regimens.

In managing their pharmacy benefits, MCOs are just starting to implement report cards that measure how pharmacists in their networks are counseling and interacting with customers. The results can be compared to national baseline parameters to help MCO pharmacy benefits managers evaluate whether certain pharmacy providers should stay in the network.

Clearly, pharmacists' ability to evaluate and monitor medication use needs to be appraised, as does customer satisfaction with their services. However, pharmacists are the only members of the health care team without a formal . report card:

\section{SUPPORT FOR REPORT CARDS}

Since their introduction in 1991, report cards have provided important information on the effectiveness, cost, access, and availability of care, the use of services, and patient satisfaction with the care received. While still evolving in format and content, report cards have already demonstrated a signifi- cant, positive effect on health care quality. For instance:

$\Delta$ Report cards have helped decrease citywide costs and the average length of hospital stays in Cleveland. They have, also been credited with improving obstetrical services and lowering the rate of Caesarean sections performed in other cities. ${ }^{3}$

$\Delta$ In one California hospital, report card results led to the refinement of procedures and changes in staff assigned to treating acute myocardial infarction patients in the emergency room. ${ }^{4}$

$\Delta$ Hospitals, MCOs, and health care professionals, aware that large purchasers are relying increasingly on performance report cards in selecting their HMOs, are using report card information to develop quality improvement tools.

A Consumers and employers are actively seeking this kind of information to help them make informed health care decisions (e.g., General Motors now provides employees with information about the quality of health care plans available to them). The result has been a profound shift in plan enrollment-as much as $30 \%$-away from lower-quality, narrower-range plans to those of higher quality and greater options.

Consumer demand for useful infor-. mation continues to grow. In a 1995 Louis Harris and Associates survey of 1,081 adults, $95 \%$ of respondents indicated that the quality of plan doctors is the most important determinant of health plan selection. However, only $32 \%$ said that they were satisfied with the amount and nature of physician-specific information received from their health plans or employers. Additionally, $50 \%$ said that they would value information about consumer ratings of hospitals and physicians, as well as report cards comparing competing health plans.

Report cards currently in use tend to describe the process rather than the outcomes of care. To enhance their worth, future report cards will have to define what is or is not working. Timely data should be included about the process of care, the competence of health care professionals, and "clinical efficacy," in which the care outcomes are measured by asking patients how they feel about their experiences. Standardization is a key factor. Thus, benchmark statistics should be emphasized to enable comparisons among health care professionals. ${ }^{6}$ These improvements should help ensure that consumers and health care purchasers can glean useful information from report cards.

\section{PHARMACY REPORT CARDS}

Demand for high-quality pharmaceutical care and standardized, useful information on community pharmacy performance will continue to rise for a number of reasons. First, new medications entering the market have increased payors' need to manage drug-utilization costs. Second, the escalating problem of medication noncompliance has heightened the need to optimize drug therapy and improve patient outcomes. Third, patient/pharmacist communication is essential to understanding not only what patients want from pharmacists, but also what they need.

However, the only tools currently available to evaluate pharmacies use cost-based measures. PBMs monitor generic substitution rates, but patient compliance for selected medications per store, which may provide an excellent indication of patient counseling, is usually overlooked.

There is, however, evidence of growing interest in compliance data. New HEDIS 1999 Behavioral Health Measures, for example, require monitoring of compliance with antidepressant therapy. MCOs and PBMs can use this information to improve the quality of their networks and help their organizations meet HEDIS requirements.

The time has come to evaluate pharmacy performance from a service perspective and examine specific characteristics of pharmaceutical care. Simply 
measuring overall satisfaction may not reveal opportunities to improve service quality. This observation is based in part on the Response-Oriented Patient Evaluation Survey (ROPES), in which pharmacy patients were asked about specific characteristics of the services they received. Despite expressing overall satisfaction with their pharmacy experience, recent ROPES respondents expressed frustration with several areas of pharmacy service: wait time, the availability of after-hours drug information, and restrictions in selecting a pharmacy. ${ }^{7}$ ROPES, therefore, helped identify areas in which quality could be advanced, just as a comprehensive report card would.

Although many satisfaction surveys primarily assess general services, more recent surveys are expanding to include pharmaceutical care. One large West Coast health plan has used a pharmacy satisfaction survey with five domainskey indicators, overall satisfaction, benefit design/program satisfaction, disease/ medication management satisfaction, and dispensing function satisfaction-to improve the quality and quantity of general and pharmaceutical care services provided in its pharmacy network. This type of broad-reaching survey represents a trend that should continue as payors mandate report cards for pharmacy.

Disease states specified in the pharmaceutical care assessment portion of this survey include hyperlipidemia, asthma, migraine headaches, diabetes, cardiovascular disease (including hypertension), depression, and anxiety. Members are questioned about how often pharmacists monitor their disease state, such as blood pressure monitoring for hypertensive patients and glucose monitoring for diabetics-services for which pharmacists are beginning to request reimbursement. Furthermore, counseling on both the disease state and the prescribed medication are assessed. Members are asked to quantify how often pharmacists actively counsel and how often they provide written materials.

Clearly, PBMs need to implement pharmacy provider network report cards.
These evaluations should examine the extent to which a pharmacist educates and counsels patients, providers, and health plans on quality care and pharmaceutical alternatives. Report cards will present pharmacists with opportunities to both improve the quality and quantity of care provided to patients and substantiate the value of pharmacists as part of the health care team.

\section{PBM RESPONSE}

In order to assess the current PBM environment and evaluate future opportunities and trends, Bimark Healthcare Communications interviewed directors of four selected major PBMs, each covering approximately 4.4 million lives, with an average of 38,000 pharmacy providers. The PBM directors all agreed that the means to assess the quality of care provided by network pharmacies and the impact of such services on outcomes are virtually nonexistent, and that progress in this direction is slow.

The PBM directors articulated a clear need for report cards to evaluate the quality of pharmaceutical care delivered. They reported that pharmacies are selected for inclusion in their networks based on geographical demand, ease of access for customers, and reimbursement requirements, without consideration for quality of care. Likewise, fee structures are influenced not by clinical services but primarily by the rate of generic substitution, another cost factor.

Responses to interview questions-specifically targeting the extent and nature of interactions between pharmacists and patients are especially telling. Only one PBM director stated that the health care organization he represents requires pharmacists to provide pharmaceutical care services beyond those mandated by state and federal regulations. The criteria used by plans to evaluate pharmaceutical care services should include, first and foremost, patient satisfaction surveys, plus pharmacist documentation, therapeutic substitution information, and compliance data that help
PBMs analyze the cost of care, but not the quality.

Patient satisfaction surveys, while a step in the right direction, are notoriously deficient in measuring cognitive services. While the surveys used by these PBM directors all inquired about the "friendliness" of support staff and whether the pharmacist adequately explains the purpose of the medication, only one organization assessed whether. the pharmacists in its network discuss therapy duration and expected side effects with patients. Again, patient satisfaction surveys typically place a much greater emphasis on whether the pharmacy is conveniently located and the waiting time is tolerable for customers. Thus, convenience factors supercede pharmaceutical care delivery.

None of the PBM directors interviewed offered specific continuing education courses for network pharmacists, a disappointing trend that reinforces the traditional role of the pharmacist as merely a dispenser of medications. Howver, with the implementation of pharmacy report cards, continuing education offers exciting opportunities for organizations to strengthen areas within their network that require improvement. As patient care and outcomes improve, overall cost of disease management should decline. Data suggest this to be true in diabetes and asthma, due to decreased long-term sequellae and emergency room visits, respectively.

PBMs have yet to recognize the value that clinical training of pharmacists adds to the resources of the health care team and, ultimately, to patient care. Such training serves to decrease the overall cost of disease management. Unfortunately, pharmaceutical benefits are carved out in such a way' that cost to PBMs means drug costs, making improved drug utilization a difficult concept to sell. When asked whether network inclusion requirements are likely to become more stringent, three PBM directors replied yes, though the quality of clinical interventions was not mentioned as a reason. Only one PBM director said that his 
Continued from page 180

organization plans to increase reimburse ment to pharmacists for additional cognitive services; two other PBMs stated that this idea is "under consideration."

The key pharmaceutical "quality issues" that PBMs felt should be monitored more closely were administrative and operating communications and formulary management compliance, both of which are associated with cost. The PBMs identified cost containment as by far the biggest issue in management of pharmacy benefit today; the greatest opportunity was seen, again, in cost control and in its demonstration of overall value.

Although pharmaceutical care is the defining philosophy in pharmacy practice today, pharmacies are financially rewarded for cost-saving programs, such as generic substitution and therapeutic switching, but rarely for cognitive services. Therefore, no financial incentives currently exist for network pharmacies to provide pharmaceutical care services; pharmacies that provide such services have little inducement to assess that care. Information from pharmacy report cards would yield the outcomes data needed to justify pharmacy reimbursement for cognitive services.

\section{CONCLUSION}

Market forces drive the health care industry, causing the purchase of care to be based principally on value - the best possible outcome at the best possible price. In this regard, pharmacy report cards can help health care purchasers make intelligent, informed decisions.

The underlying goal of report cards. is to place greater accountability on health care providers for the quality of services provided. A report card on pharmacy performance should show how well pharmacists in a given plan educate and counsel patients and health care professionals on treatment regimens and medical alternatives. As they have with health systems, outcomes data might motivate the retail pharmacy sector to improve the quality and range of services. This outcome would create a win/win scenario for health care providers, health care managers, payors, and patients alike.

\section{$\Delta \quad$ References}

1. O'Malley C. Quality measurement for health systems: accreditation and report cards. Am J Health-Syst Pharm 1997; 54: 1528-35.

2. Beed $G$. The value of information and HEDIS measures. Special Report: Second Annual

Managed Care 2000. East Hanover, N]: Novartis Pharmaceuticals Corp 1997: 4-5

3. Nash DB. Report on report cards. Health Policy Newsletter, Jefferson Health System 1998; 11: 1-2.

4. Rainwater JA, Romano PS, Antonius DM. The California Hospital Outcomes Project: how useful is California's report card for quality improvement? J Quality Improvement 1998; 24: 31-39.

5. Managed Care Communications Inc. Part Il: Who are the truest arbiters of quality? Quality in healthcare: issues, controversies \& solutions. Boston, MA: Managed Care Communications Inc. 1997: 5-12.

6. Kaplan JG. The report card's role in health care decision-making: Part I. Managed Care Interface 1997(Dec): 61-65.

7. Kucukarslan SN, Pathak DS, Summers K. Response-oriented patient evaluation survey (ROPES): an administrator's tool for identifying opportunities for service quality improvement. J Managed Care Pharmacy 1998; 4: 311-20. 